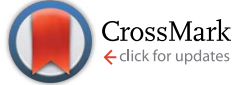

Cite this: RSC Adv., 2014, 4, 62362

Received 22nd August 2014 Accepted 3rd November 2014

DOI: $10.1039 / c 4 r a 09089 f$

www.rsc.org/advances

\section{A novel hybrid material: an inorganic silica aerogel core encapsulated with a tunable organic alginate aerogel layer $\dagger$}

\author{
Zeynep Ulker and Can Erkey*
}

A novel layered material consisting of a silica aerogel core encapsulated by an alginate aerogel layer was developed. The components of the hybrid aerogel had the high surface area and high porosity of pure aerogels which should lead to development of new layered systems for a wide variety of applications.

Over the past few decades, nanostructured materials have shown a great promise in a variety of application areas like catalysis, insulation, sensors or drug delivery. ${ }^{1-3}$ Among them, nanoporous aerogels were found to be promising materials for the encapsulation and adsorption of a variety of molecules due to their large inner surface areas, high surface to volume ratios, large pore volumes and uniform pore sizes. ${ }^{4-6}$ The development of a multi layered material based on aerogels should provide new opportunities for applications such as increased and/or controlled loading/adsorption and controlled release. Moreover, the possibility of tuning the properties of the layers by controlling the preparation conditions should make these materials very attractive for a wide variety of applications. There have already been several attempts to synthesize layered structures based on different porous materials such as hydrogels. Ladet et al. prepared the first multi layered hydrogel from the periphery towards the core by controlling the kinetics of the gelation process. ${ }^{7}$ Dai et al. developed a multi layered hydrogel structure with and without internal space between separate layers by a precise control of the processing conditions via a dynamic self assembly method. ${ }^{8}$ They suggested that a layered structure from the core to the surface might facilitate the drug loading and cell immobilization. Along similar lines, Duan et al. synthesized a new multi layered hydrogel using agarose gel-core as the template by sequential cross-linking. ${ }^{9}$ They showed that the layer formation was dependent on the type of cross-linker, its concentration and/or soaking time. Alternatively, layered

Koç University, Rumelifeneri Yolu, 34450 Sarıyer, Istanbul, Turkey. E-mail: cerkey@ ku.edu.tr; Tel: +902123381866

$\dagger$ Electronic supplementary information (ESI) available. See DOI: $10.1039 / \mathrm{c} 4 \mathrm{ra} 09089 \mathrm{f}$ aerogel based materials such as silica aerogel coated with PEG hydrogel $^{\mathbf{1 0}}$ or with polymeric materials ${ }^{\mathbf{1 1}}$ were also prepared. However, to our knowledge there are no reports in the literature of a layered material prepared from two distinct aerogels with high surface areas and porosities. This approach may lead to development of new materials with desirable properties for different applications. Two different compounds can be incorporated with high loadings in a single material. Loading of these different compounds on separate layers could prevent their undesirable interactions. Moreover, it may be possible to use layered materials for sequential release of two different compounds.

In this study, we designed and prepared a new layered aerogel system comprising a biocompatible silica aerogel core and a biodegradable alginate aerogel layer while retaining their porous characteristics intact. The alginate aerogel layer was shown to protect the hydrophilic silica aerogel core when immersed in liquid solutions which otherwise collapses easily. It was also demonstrated that the kinetics of drug release was changed due to the existence of a thin alginate aerogel layer around the silica aerogel core.

Silica aerogel was prepared by the conventional two step solgel synthesis and the alginate aerogel was synthesized using the internal setting method. ${ }^{12}$ The alginate layer formation was triggered by the diffusion of cross-linking ions from the silica alcogel core into a sodium alginate solution in which it was immersed. The crosslinking ions were shown to create an electrostatic binding between macromolecules and form an egg-box structure. ${ }^{\mathbf{8} 13}$ To synthesize the silica alcogel core, the silica precursor, tetraethylorthosilicate (TEOS), was primarily hydrolyzed in a mixture of $\mathrm{CaCl}_{2}$ solution (instead of pure water) and ethanol. The rate of hydrolysis reactions was accelerated by the addition of an acid catalyst as shown in Fig. 1.

After the hydrolysis, the base catalyst was added to increase the rate of condensation reactions which eventually lead to gelation. The gelled samples which are called alcogels were then placed in Na-alginate solution. The diffusion of cross-linking, $\mathrm{Ca}^{2+}$, ions from the porous silica alcogel structure to the 


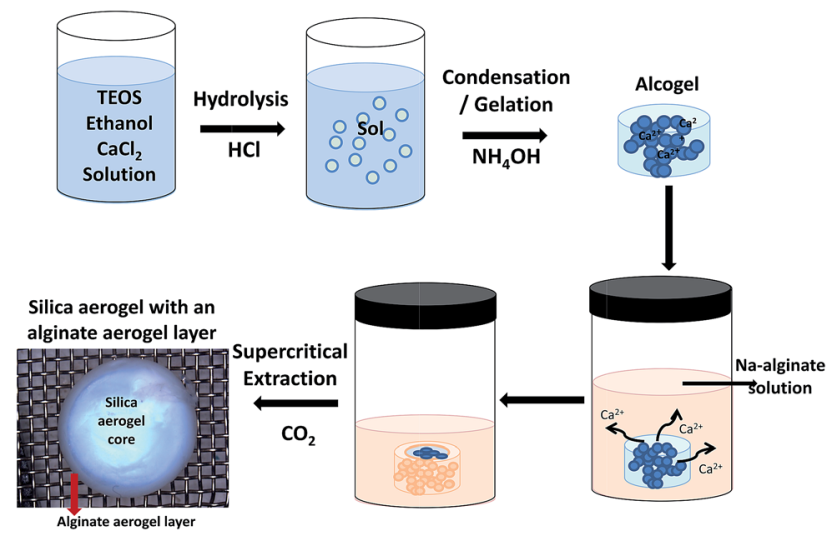

Fig. 1 The scheme for the preparation of a silica aerogel encapsulated with an alginate aerogel.
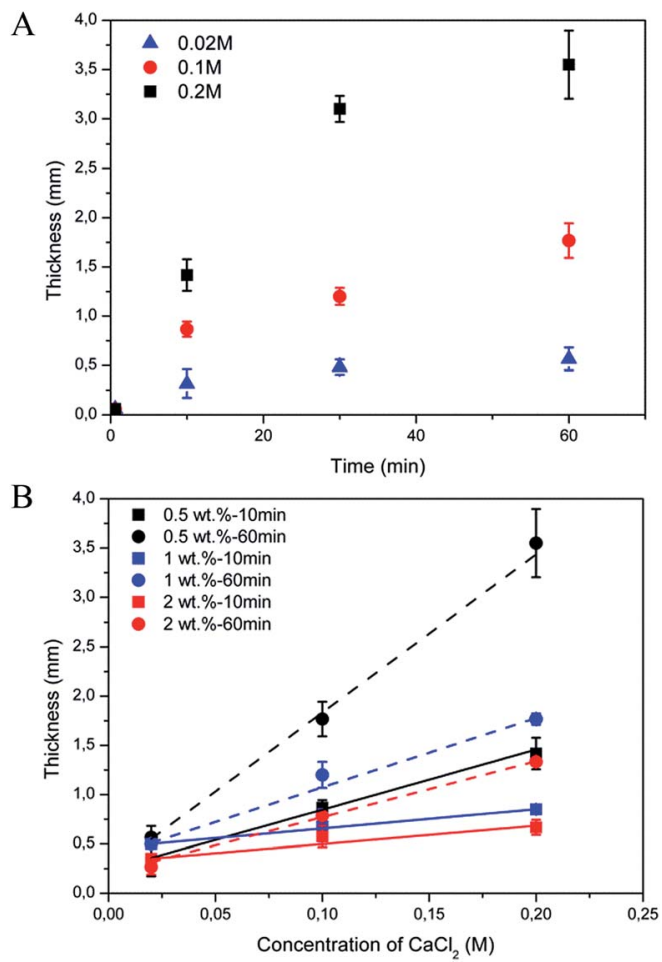

Fig. 2 (A) The change in the thickness of the alginate layer with time using different concentrations of $\mathrm{CaCl}_{2}(0.5$ wt.\% alginate). (B) The thickness of the alginate layer versus $\mathrm{CaCl}_{2}$ concentration at specific times (10 $\mathrm{min}$ and $60 \mathrm{~min}$ ).

alginate solution resulted in the formation of an alginate layer around the silica alcogel core as shown in Fig. 1. The shape of the initial silica alcogel was found to determine the final shape of the layered material due to the homogeneous diffusion of the $\mathrm{Ca}^{2+}$ ions from the alcogel core.

The growth of the alginate layer continued as long as the silica alcogel core had sufficient free $\mathrm{Ca}^{2+}$. The separation of the newly formed layered structure from the sodium alginate solution stopped the growth of the alginate layer. The resulting material was further cross-linked (or cured) in a $\mathrm{CaCl}_{2}$ solution
A

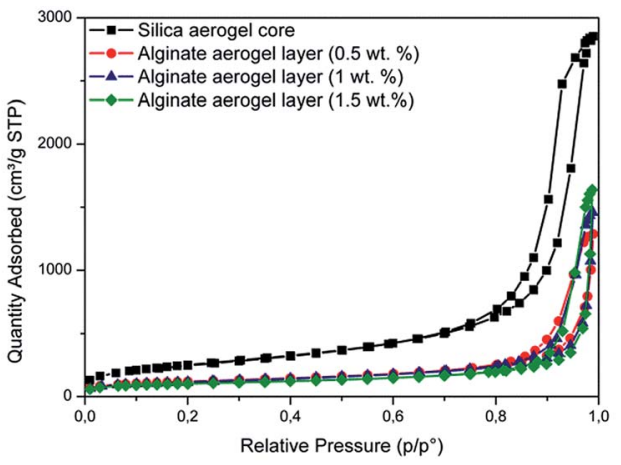

B

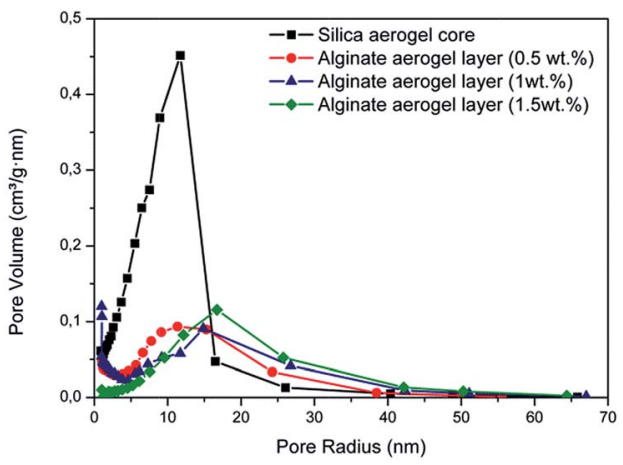

Fig. 3 (A) Adsorption isotherms of $\mathrm{N}_{2}$ on alginate aerogel layers and silica aerogel core, (B) the pore size distribution of different alginate aerogel layers and silica aerogel core.

which in turn was found to reduce the thickness of the alginate layer. ${ }^{8}$ After gelation, if the layered sample was first dipped in $\mathrm{CaCl}_{2}$ solution and then placed in a different Na-alginate solution following similar principles of dip coating for membrane formation, a new layer formed leading to a multi layered aerogel structure. Due to the difference in the rate of layer formation, two successive layers would be expected to have different pore properties. The same strategy might also be followed for the initial layer formation; however it would be more difficult to control the layer thickness. After curing, the resulting layered aerogel was subjected to successive aging steps prior to supercritical drying. The samples were successively placed in a series of ethanol-water baths with increasing alcohol concentrations $(10,30,50,70,90$ and 100). The reason of using this kind of aging was to prevent the structural changes which could have occurred if $100 \%$ ethanol was immediately added..$^{14}$ The ethanol in the pores was then removed by supercritical drying using $\mathrm{CO}_{2}$ at 90 bar and $313 \mathrm{~K}$.

As shown in Fig. 2A, the layer got thicker with increasing crosslinking time and increasing $\mathrm{CaCl}_{2}$ concentration in the pores of the silica alcogel due to the existence of more free divalent cations. The change in $\mathrm{Ca}^{2+}$ ion concentration in the silica alcogel core with a diameter of $1.13 \mathrm{~cm}$ changed the time required for the growth of the alginate layer to a certain thickness.

The thickness reached almost $4 \mathrm{~mm}$ in 60 minutes for the highest $\mathrm{CaCl}_{2}$ concentration versus $0.5 \mathrm{~mm}$ for the lowest $\mathrm{CaCl}_{2}$ concentration for experiments carried out using the same composition of alginate solution. The rate of layer growth 
Table 1 BET Results for silica aerogel core and alginate aerogel layers with different concentrations

\begin{tabular}{|c|c|c|c|c|}
\hline Sample & $\begin{array}{l}\text { BET surface area } \\
\left(\mathrm{m}^{2} \mathrm{~g}^{-1}\right)\end{array}$ & $\begin{array}{l}\text { Pore volume } \\
\left(\mathrm{cm}^{3} \mathrm{~g}^{-1}\right)\end{array}$ & $\begin{array}{l}\text { Desorption average } \\
\text { pore radius }(\mathrm{BJH})(\mathrm{nm})\end{array}$ & $\begin{array}{l}t \text {-plot } \\
\text { micropore volume }\left(\mathrm{cm}^{3} \mathrm{~g}^{-1}\right)\end{array}$ \\
\hline Silica aerogel core & 905 & 4.1 & 8.0 & 0.02 \\
\hline Alginate aerogel layer $(\% 1 \mathrm{w} / \mathrm{w})$ & 400 & 0.9 & 11.7 & 0.031 \\
\hline Alginate aerogel layer $(\% 0.5 \mathrm{w} / \mathrm{w})$ & 407 & 1.1 & 10.3 & 0.036 \\
\hline
\end{tabular}

decreased with increasing time which can be attributed to the reduced rate of diffusion of $\mathrm{Ca}^{2+}$ ions through the silica alcogel and then the newly formed alginate hydrogel layer. As seen in Fig. 2B, there was a linear correlation between the concentration of $\mathrm{Ca}^{2+}$ ions and the thickness of the alginate layer at a certain time for a fixed alginate concentration. As the initial $\mathrm{CaCl}_{2}$ concentration increased, the thickness of the alginate layer increased at a constant alginate concentration due to the existence of more free divalent cations. Moreover, as the alginate concentration was increased, the growth rate of the layer was reduced at a constant $\mathrm{CaCl}_{2}$ concentration due to the formation of more cross links creating a tighter layer. This linear relation enables the selection of the appropriate concentration for a desired thickness over a specified time period.

The pore properties of the samples were characterized using $\mathrm{N}_{2}$ physisorption. The samples were primarily degassed at $60{ }^{\circ} \mathrm{C}$ under vacuum prior to analysis. The effect of increasing Naalginate weight ratio in the solution on the pore properties of the alginate aerogels was also investigated. The pore structure was then analyzed with Zeiss Ultra Plus Field Emission Scanning Electron Microscope after gold coating. The average pore size and pore size distribution of the samples obtained from BET analysis were consistent with SEM images.

$\mathrm{N}_{2}$ physisorption results as shown in Fig. 3A and B indicated that the formation of an alginate layer around the silica alcogel did not significantly affect the pore characteristics of the silica aerogel. The small reduction in its surface area (which is usually around $\sim 950-1000 \mathrm{~m}^{2} \mathrm{~g}^{-1}$ ) could be related to its volume shrinkage during the layer formation due to the absence of the conventional aging step which is usually carried out to strengthen the network. In addition, the surface area of alginate aerogel layers was sufficiently high indicative of possible high loading capacities. Furthermore, the difference in pore sizes could be used to enable the control over the loading and release of different sized, especially large biomolecules.

As shown in Table 1, the surface areas of alginate aerogel layers which were prepared with different concentrations ranging from $0.5 \mathrm{~g}$ of alginate per 100 grams of water to $1.5 \mathrm{~g}$ of alginate per 100 grams of water were found to be similar ${ }^{14,15}$ and even higher ${ }^{16}$ compared to the values in the literature for alginate aerogels. Veronovski et al. showed that increasing the concentration of the alginate solution resulted in a higher degree of cross-linking leading to more compact and stable gels. ${ }^{14}$ Therefore, increasing the concentration of the polymeric chains was expected to create smaller pores and thus reduced pore volumes. Even though the data given in Table 1 are in
A

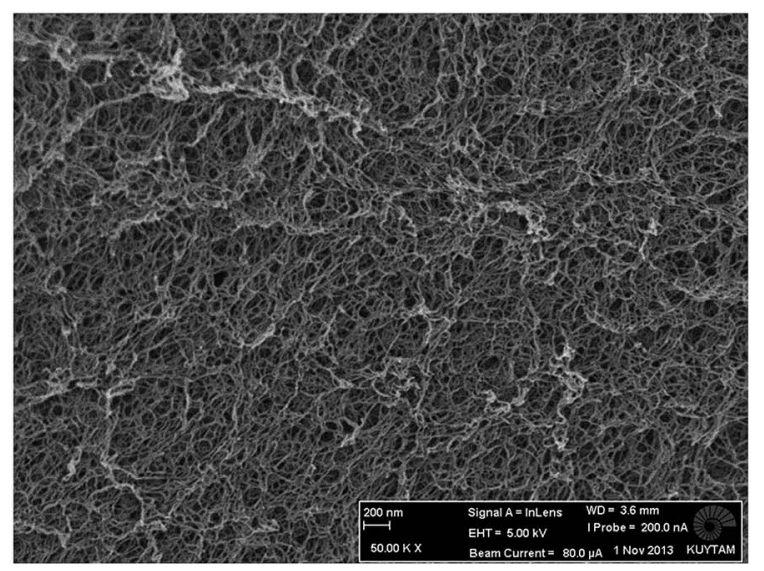

B

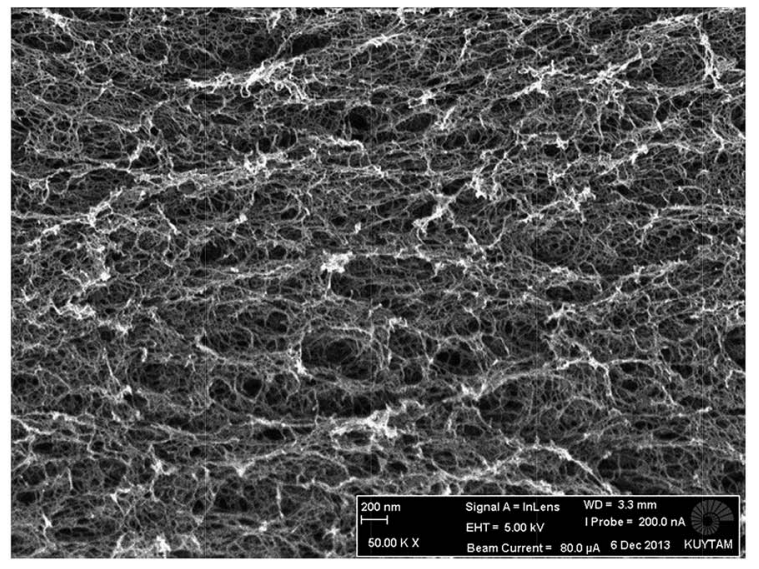

C

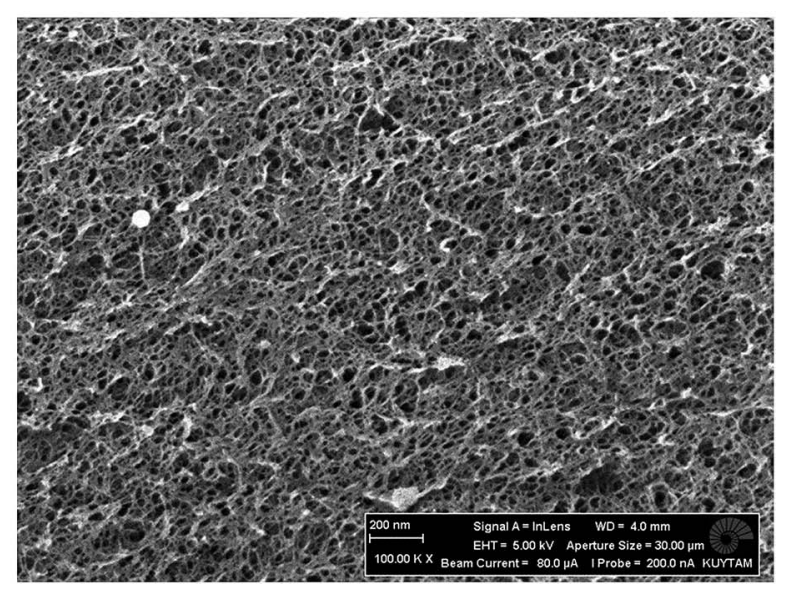

Fig. 4 (A) SEM image of the alginate aerogel layer prepared with $\% 0.5$ w/w Na-alginate in water, (B) SEM image of the alginate aerogel layer prepared with $\% 1 \mathrm{w} / \mathrm{w} \mathrm{Na}$-alginate in water, (C) SEM image of the alginate aerogel layer prepared with $\% 1.5 \mathrm{w} / \mathrm{w} \mathrm{Na}$-alginate in water. 


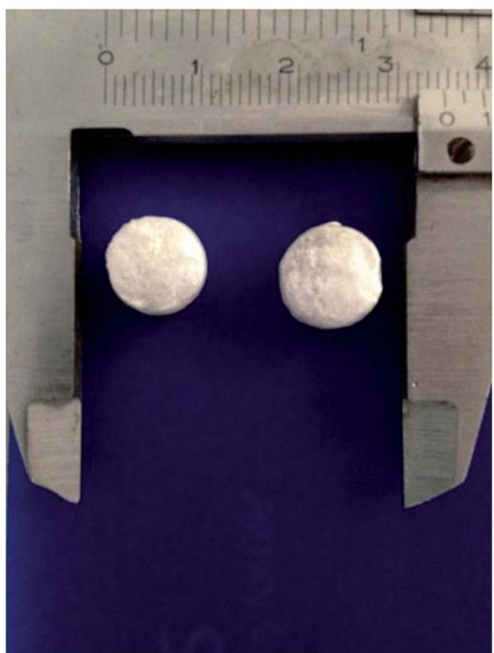

Fig. 5 Paracetamol loaded silica aerogels encapsulated with alginate aerogel layers (hybrid aerogel at the right was synthesized after 4 months the hybrid aerogel at the left was synthesized).

agreement with this hypothesis, the magnitude of the difference among the pore volumes is smaller than expected. This can perhaps be attributed to a higher degree of cross-linking which leads to a higher resistance to shrinkage during supercritical drying due to the higher strength of the solid network. Moreover, the presence of smaller pores of the alginate aerogel layers compared to the silica aerogel core could enable the use of this system for molecules with different sizes.

As shown in Fig. 3B, alginate aerogel layers had wide pore size distributions. This can also be seen from the SEM images of the alginate aerogel layers which were prepared with different concentrations of Na-alginate as shown in Fig. 4A-C. SEM images also indicate that the alginate aerogel layers have a fibrous cross-linked structure with different size pores. From the average pore size and specific surface area data, the alginate aerogel layers can be considered to be mesoporous with a small fraction of microporosity compared to the silica aerogel which has a smaller micropore volume. The increasing initial alginate

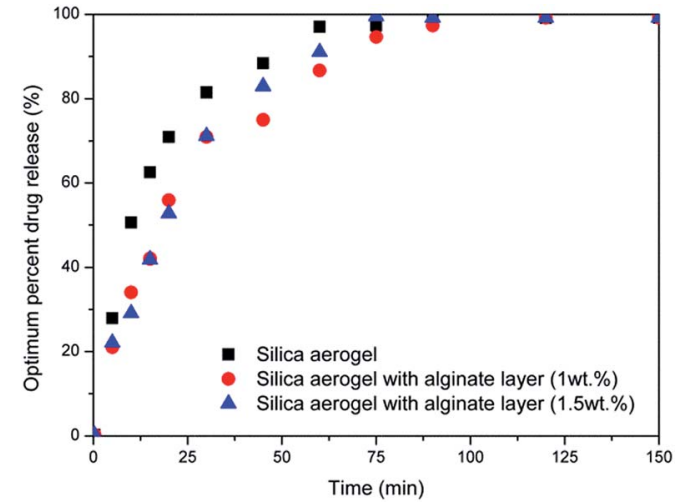

Fig. 6 The release profiles of paracetamol in PBS buffer with a pH of 5.8 . concentration also resulted in a slight increase in micropore volume as shown in Table 1.

To show the effect of the alginate aerogel layer on the drug release kinetics, a layered system with paracetamol as the model drug was prepared. Paracetamol was loaded to the silica alcogel phase and this matrix was then covered with an alginate layer (see $\mathrm{ESI} \dagger$ for the details of the procedure). The drug loaded hybrid aerogels were shown to be stable even after 4 months of their synthesis as shown in Fig. 5 .

The release of paracetamol from a native silica aerogel and silica aerogels encapsulated with alginate aerogel layer was compared in PBS buffer (pH: 5.8). Nanodrop 1000 spectrophotometer was used to monitor the release of the paracetamol at $244 \mathrm{~nm}$. The alginate aerogel layer prevented the collapse of the silica aerogel when contacted with the buffer solution, swelled with time, and thus reduced the burst drug release and slightly retarded the drug release as shown in Fig. 6. As the thickness of the alginate aerogel layer was around 1-2 $\mathrm{mm}$, the release was not significantly affected but comparably slowed down indicating that these hybrid aerogels are promising as drug delivery vehicles. Increasing the concentration of the alginate in the initial solution and increasing the thickness of the layer would lead to a slower release. Thus, more work will be carried out in order to analyze the effect of the thickness of alginate aerogel layers and the concentration of the alginate in the layers on the drug release kinetics.

\section{Conclusions}

A novel layered material consisting of two distinct aerogels was prepared for the first time. The approach of combining different porous structures in one single material is expected to widen the application area of these materials especially in biomedical field for controlled release applications. The layers were not only shown to protect the core and to slightly retard the release of the drug but are also expected to be used as a delivery device capable of carrying different molecules at the same time for sequential release.

\section{Notes and references}

1 M. Goldberg, R. Langer and X. Jia, J. Biomater. Sci., Polym. Ed., 2007, 18, 241-268.

2 F. Zaera, Chem. Soc. Rev., 2013, 42, 2746-2762.

3 Z. Ulker and C. Erkey, J. Controlled Release, 2014, 177, 5163.

4 I. Smirnova, S. Suttiruengwong and W. Arlt, J. Non-Cryst. Solids, 2004, 350, 54-60.

5 C. A. García-González, M. Alnaief and I. Smirnova, Carbohydr. Polym., 2011, 86, 1425-1438.

6 S. Bozbag, S. Kostenko, M. Kurykin, V. Khrustalev, A. Khokhlov, L. Zhang, M. Aindow and C. Erkey, J. Nanopart. Res., 2012, 14, 1-13.

7 S. Ladet, L. David and A. Domard, Nature, 2008, 452, 7679.

8 H. Dai, X. Li, Y. Long, J. Wu, S. Liang, X. Zhang, N. Zhao and J. Xu, Soft Matter, 2009, 5, 1987-1989. 
9 J. Duan, R. Hou, X. Xiong, Y. Wang, Y. Wang, J. Fu and Z. Yu, J. Mater. Chem. B, 2013, 1, 485-492.

10 S. Giray, T. Bal, A. M. Kartal, S. Kizılel and C. Erkey, J. Biomed. Mater. Res., Part A, 2012, 100, 1307-1315.

11 M. Alnaief, I. Smirnova, S. Antonyuk, S. Heinrich and S. Roth, Chem. Ing. Tech., 2010, 82, 1466-1467.

12 C. M. Silva, A. J. Ribeiro, I. V. Figueiredo, A. R. Gonçalves and F. Veiga, Int. J. Pharm., 2006, 311, 1-10.
13 M. Robitzer, L. David, C. Rochas, F. Di Renzo and F. Quignard, Langmuir, 2008, 24, 12547-12552.

14 A. Veronovski, Ž. Knez and Z. Novak, J. Supercrit. Fluids, 2013, 79, 209-215.

15 R. R. Escudero, M. Robitzer, F. Di Renzo and F. Quignard, Carbohydr. Polym., 2009, 75, 52-57.

16 T. Mehling, I. Smirnova, U. Guenther and R. H. H. Neubert, J. Non-Cryst. Solids, 2009, 355, 2472-2479. 